\title{
A Future of Traffic Management Toward a Hybrid System of Roadside and Personal Mobile Devices
}

\author{
Tom Thomas \\ Center of Transport Studies, University of Twente, p.o.box 217, 7500 AE Enschede, The Netherlands \\ t.thomas@utwente.nl
}

Keywords: Travel Time, Travel Demand, Urban, Prediction, Detection Loops, GPS.

\begin{abstract}
In the last few decades, increasing traffic has led to serious problems in urban areas. Travel times for travellers have increased and the liveability in residential areas has declined due to pollution and issues concerning safety. Whereas new road and rail infrastructure in densely populated areas is costly, a better utilisation of existing infrastructure appears to be more attractive. This becomes increasingly possible due to huge developments in the world of Information and Communication Technology (ICT). However, implementing ICT solutions for the purpose of traffic management remains challenging. First, it is quite a task to set up a system in which infrastructure, travel vehicles and travellers can communicate with each other. Secondly, it is quite difficult to gather and exchange traffic and travel information in such a way that the traffic situation improves significantly. This paper deals with the latter issue, and it provides an outline for the possible architecture of a future traffic management system. It concludes that in such a system both mobile devices, like smartphones and navigation systems, and roadside devices, like loop detectors and cameras, need to be included to arrive at optimal results.
\end{abstract}

\section{INTRODUCTION}

Congestion has increased significantly in the last few decades. The efficient use of existing infrastructure by dynamic traffic management (DTM) is one of the strategies to reduce congestion and related problems like air pollution. An important requirement is the availability of detailed traffic information such as travel demand and travel times.

In the Netherlands and many other developed countries, highway data are collected by a high concentration of detection loops which yield information on traffic intensities and travel times. In urban areas traffic information is much scarcer and only since quite recently, traffic data gathered by roadside devices like detection loops have become available in traffic information centres (e.g., Hasberg and Serwill, 2000, Kellerman and Schmid 2000, Leitsch, 2002). For urban areas, the traffic circulation is usually estimated by a combination of intensity measurements and traffic models. However, with new measurement methods by which individual vehicles are identified (Blokpoel and Vreeswijk, 2011), accurate roadside measurements of travel times and routes will become possible.
At the same time, the use of mobile sensor data such as GPS and GSM has been increasing rapidly, which have led to separate travel time estimators (e.g., Google Traffic, 2013). Because these data are gathered by personal devices such as navigation systems and smartphones, travel information and advice can be personalized and adapted to the preferences of the individual traveller (e.g., Bie et al., 2012). In case of smartphones, travel information does not have to be limited to car trips, but may also include several other travel modes.

Roadside and mobile sensor data techniques both have their specific strengths. In this paper, a possible architecture for future DTM is put forward in which both data sources are combined at some point. In section 2, the requirements for a future DTM system are provided. Then, in section 3 and 4 , the advantages, disadvantages and possibilities of mobile and roadside sensor data are described respectively. Based on the requirements for a future DTM system and the strengths and weaknesses of roadside and mobile sensor data, section 5 describes an outline for the architecture of a possible future DTM system. 


\section{DTM REQUIREMENTS}

The requirements for a modern DTM system should be based on two pillars. First, the traffic system, usually the responsibility of a traffic manager, should run smoothly. This may mean several things, but in general the objective is to minimize the total delay on the (road) network and to minimize external costs caused for example by traffic accidents or air pollution. Secondly, the traffic users, i.e. travellers, want to travel smoothly. This may also mean various things for individual travellers, but in general travellers want to minimize their (perceived) travel time and cost while perceiving the journey as being safe and comfortable.

In other words, the traffic manager's objective is to optimize the traffic system as a whole, while travellers want to optimize their individual journeys. Both things are not necessarily resulting in the same traffic equilibrium, and in some cases they are even clearly conflicting with each other. One of the main challenges of a modern DTM is therefore to reconcile the two.

Meeting the objectives of the traffic manager and individual traveller also requires different information needs. An individual traveller needs to have traffic information about the possible travel modes and routes that are relevant to him, i.e. those that can be used to reach the preferred destination. Detailed information only needs to be available during the time of traveling. This is by no means trivial, because it still requires a prediction of the traffic situation in the near future, i.e. until the journey is expected to be completed.

However, this task is relatively easy compared to optimizing management objectives. Whereas the individual traveller is merely influenced by surrounding traffic, the traffic manager is influencing traffic itself, which effects are much harder to predict. Moreover, the effects of traffic operations like traffic lights are not necessarily instantaneous, but may show delays. For example, a traffic measure in one part of a city can have an effect in another part half an hour later. In the ideal case, the traffic situation should therefore be predictable for the whole network, for different traffic management scenarios, and during a longer period of time, for example a whole peak hour.

Fulfilling the needs of individual travellers on the other hand has its own challenges. While there is one traffic manager with one set of objectives, there are many individual travellers, all with their own perceptions, preferences and habits that play an important role in the decision making process. In this modern individual oriented society, traffic information and travel advice of one fits all is becoming less acceptable. By using individual devices like smartphones, it is also becoming technically possible to provide personalized traffic information and travel advice.

From the aforementioned considerations, one can arrive at the following requirements:

1. Traffic management requires accurate predictions about the traffic situation for the whole network, different traffic management scenarios and whole (peak) periods.

2. Individual travellers need personalized multimodal travel advice based on their preferences and habits.

3. A traffic management measure should not lead to the perception of travellers that they are worse off due to the measure or are harmed unfairly by it.

The third requirement tries to reconcile differences between the interests of traffic manager and individual travellers. Of course, it is impossible to satisfy all travellers. However, it might be possible to introduce measures such that travellers do not notice they are worse off and therefore do not change their behaviour, or such that travellers do not perceive the alternative they switch to as worse or unfair.

\section{MOBILE SENSOR DATA}

Mobile sensors like GPS and GSM are widely used in smartphones and navigation systems nowadays. Initially, they were used for navigation, but as their numbers increase, they are now also being used for estimating travel times on road trajectories (e.g., Google Traffic, 2013). There are however more applications: they can reveal travel patterns of individuals and groups of travellers.

In most countries, including the Netherlands, the understanding of people's travel behaviour is based on cross-sectional travel surveys where in general only one day is surveyed for each respondent in representative periods (Ortuzar et al., 2010). From these data, origin destination matrices, modal split (mode choice) and route choice are estimated and used in models that model urban traffic flows.

However, this is not enough to gain a proper understanding of the dynamics in travel behaviour. More specific, cross-section data do not give any information to ascertain how choices will vary over 
time (i.e. policy response) if the system changes. Studies with GPS-devices show a strong variation in multi-day travel behaviour (e.g., Stopher amd Zhang, 2011). People are shown to visit new places even after several months of monitoring (Schönfelder and Axhausen, 2010). Apart from determining destination and mode choice patterns over longer periods of time, GPS data are increasingly used to study route choice (e.g., Jan et al. 2000, Zhu and Levinson 2009, Papinski and Scott 2011).

Derived from standard economics it is often assumed in transport modeling that travelers are rational decision makers and have perfect knowledge on all available choice alternatives. There is increasing recognition that these assumptions are debatable. In reality, people may have limited knowledge and constrained cognitive abilities, leading to prejudiced reasoning and seeming randomness in choice behavior (e.g. Avineri and Prashker, 2004). This has been described as bounded rationality or satisficing behavior, first introduced by Herbert Simon (Simon, 1955), and also found its way into transportation research (Mahmassani and Chang, 1987, Jayakrishnan et al., 1994). Since then, multiple studies suggested that these irrational behaviors are neither random nor senseless; they are systematic, consistent, repetitive, and therefore predictable (Tversky and Kahnemann, 1981, Ariely, 2009).

A well-known mechanism derived from the principles of bounded rationality is the notion of indifference band (Mahmassani and Chang, 1987). According to the theory of indifference bands, drivers will only alter their choice when a change in the transportation system or their trip characteristics, for example the travel time, is larger than some individual-situation-specific threshold.

More in general, travelers appear to make their decisions based on their perception of alternatives, which is biased according to the 'choice-supportive bias'. That is, people are more likely to attach positive feeling to options they choose and attribute negative features to options they reject (Mather et al., 2003, Henkel and Mather, 2007) even if that would be irrational. In terms of travel choices this suggests that travelers have different perceptions of options they frequently use than options they hardly use (Vreeswijk et al. 2013).

These findings may play an important role in future DTM, especially in fulfilling requirements 2 and 3. Although some travelers may be worse off when the overall network performance is optimized, it may be possible to choose DTM measures for which travelers do not perceive they are worse off or do not find this a problem. This will only be possible, however, when travelers get personalized travel advice based on their preferences and habits. For this, mobile sensor data appear to be indispensable.

\section{ROADSIDE DEVICES}

The use of smartphone, carrying among others a GPS-sensor, will probably rise in the coming years, enabling new data acquisition opportunities (Stopher, 2009, Nitsche et al., 2012). In addition, there already are numerous smartphone applications gathering personal travel data (e.g. UbiActive (Fan et al. 2012), Trip Analyzer (Li et al., 2011), and tripzoom (Bie et al., 2012)). Finally, smartphones or navigation devices are used as probes to estimate travel times on main roads. The question thus arises whether roadside devices are still necessary in the future.

To answer this question, we need to consider requirement 1 from section 2: "Traffic management requires accurate predictions about the traffic situation for the whole network, for different traffic management scenarios and over whole (peak) periods".

This requirement implies several things at the same time. First, information is needed on the traffic situation. This is much more than travel time alone. Policy makers are not only responsible for travellers, but also for the environment that is harmed by traffic. Pollution, noise hindrance and safety are important external factors which need to be considered, especially in dense residential areas or near locations that attract vulnerable groups such as schools. This implies that certain vulnerable, busy or economically important areas, locations or corridors, may need to be monitored continuously. Because many external effects depend on traffic intensity, this important quantity should be included in the monitoring.

Secondly, predictions are required for the whole network under various (possible) management scenarios. This implies that traffic intensities and travel times should be predictable when the traffic manager decides to increase or reduce the capacity of certain roads (for example by giving more or less green time). Because travel time shows a strong nonlinear dependence on network intensities (demand) and capacities (supply), it is difficult to predict travel time when intensities and capacities are unknown, especially when small changes in intensity have a 
large effect on travel time. This is actually the case when it matters (i.e. in urbanized areas with a lot of traffic), while at the same time intensities are known to show strong variation within days and between days (Thomas et al., 2008). Accurate travel time predictions under varying (management) scenarios are therefore only possible when network demand and supply are predictable.

From this, it can be concluded that traffic management requires continuously monitoring of travel times, traffic intensities and capacities throughout the network or at least at, in or along important locations, areas or corridors.

At the moment this is not possible with mobile sensors. Mobile sensor (GPS) samples for public use are simply much too small. This may be changing (e.g. Rieser-Schüssler et al. 2012) somewhat, but the expectation is that, in general, public GPS samples will remain limited, (partly) due to privacy restrictions and commercial interests. In other words, large amounts of mobile sensor (GPS) data may remain out of reach for traffic management. Even if mobile sensor data would increase substantially for public use, there will always be some travelers missing from the data. For example, commuters will be less inclined to use navigation. Therefore, it can be questioned whether there will ever be enough mobile sensor (GPS) data to monitor traffic intensities and capacities of the important road sections with enough accuracy.

Roadside observations can fill this gap. In urban areas, single detection loops have long been used to measure occupation levels and intensities as input for traffic light operations. Network monitoring is more difficult with these data, because delays cannot easily be estimated in saturated conditions (when queues form near traffic lights), and individual vehicles cannot be followed through the network. As a result these measurements don't provide information on OD patterns and routes.

However, this is changing due to increasing use of cameras and new induction detection techniques that enable the identification of individual vehicles (Blokpoel and Vreeswijk, 2011). Thus, with these roadside devices located at important intersections, travel times, intensities and capacities can be measured directly throughout the network. Together with prediction algorithms like neural networks (e.g., Dharia, and Adeli, 2003, Yin et al. 2002), pattern matching models (e.g., Bajwa et al., 2004), extrapolation models (e.g., Wild , 1997, Chrobok et al., 2004, Thomas et al., 2009) or clustering models (e.g., Chung, 2003, Weijermars, 2007), more accurate traffic predictions of intensities and travel times will then become possible given certain management scenarios.

\section{SYNTHESIS}

As we have seen in the previous sections, traffic managers and travelers use different devices, i.e., roadside and mobile devices respectively, to acquire traffic information. Although mobile devises like smartphones with GPS become increasingly important, roadside devices might remain the main source of information for traffic management, because besides travel time they are able to provide accurate information on intensities and capacities.

The traditional use of traffic information by policy makers and travelers as shown in Figure 1 therefore remains quite realistic. The Figure shows two independent symmetric systems for both traffic management and the individual traveler. Both systems have objectives, i.e., policy objectives for the traffic manager and travel objectives for the traveler. By confronting these objectives with traffic and travel information respectively, traffic operations are set to manage the traffic system, and travel decisions are made to execute a trip. The traffic information is derived from data from roadside sensors, while travel information is derived from mobile sensor data.

Of course this Figure is a simplistic illustration of reality. The division between the use of roadside and mobile sensors is in reality not this strict, and a single box could in itself represent a complicated process with feedback loops. Traffic operations, for example, represent an interplay between instruments, such as traffic lights, variable message signs or route guidance panels, and traffic managers, while travel decisions may include more than only the traveller's behaviour. In fact, nowadays most travellers are assisted by travel apps that provide the traveller with information or advice. Travel apps are therefore implicitly included in travel decisions. The use of travel information in travel apps is more subtle than the figure indicates. Mobile sensor data of other users are used to provide reliable information on relevant travel modes and routes, while historical travel choices of the user may be used to personalize the advice. However, for the broader picture, these issues do not need to be considered in detail here.

The weak part of the traditional concept, as illustrated by Figure 1, is the lack of any interaction between traffic management and traveller. This leads to drawbacks regarding all three DTM requirements. 

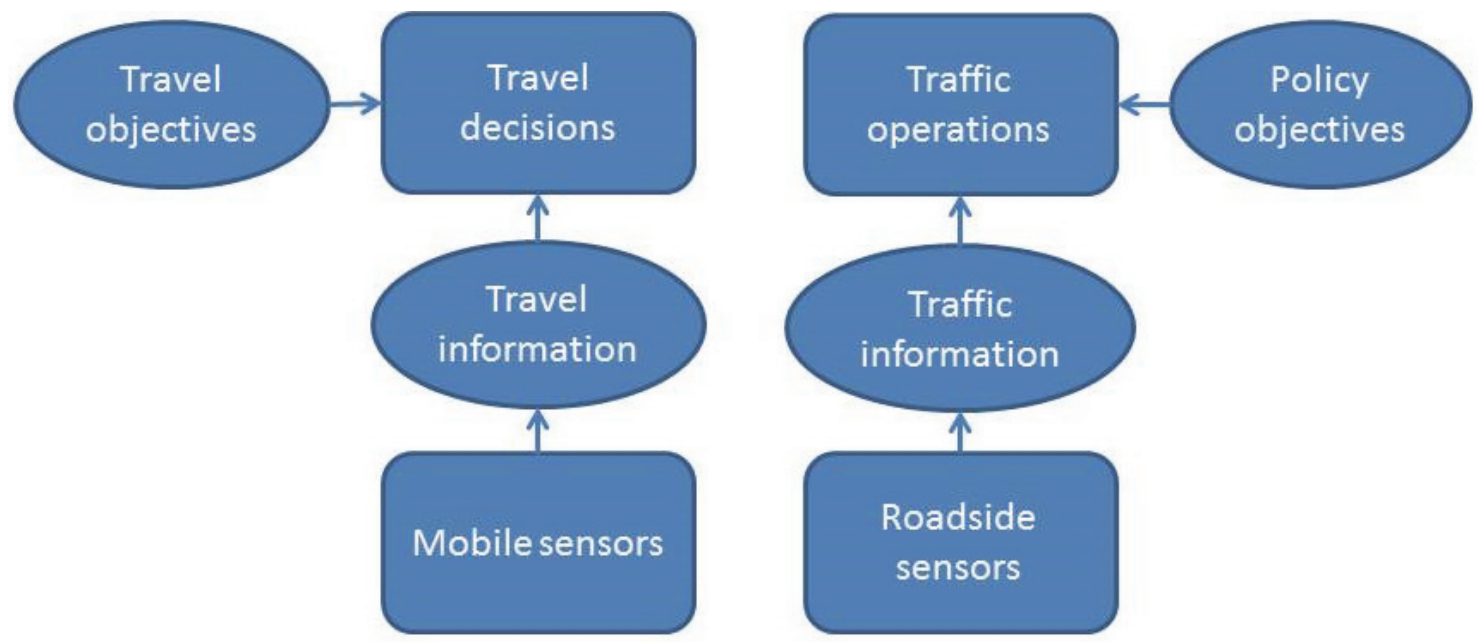

Figure 1: scheme of traditional use of mobile and roadside sensors.
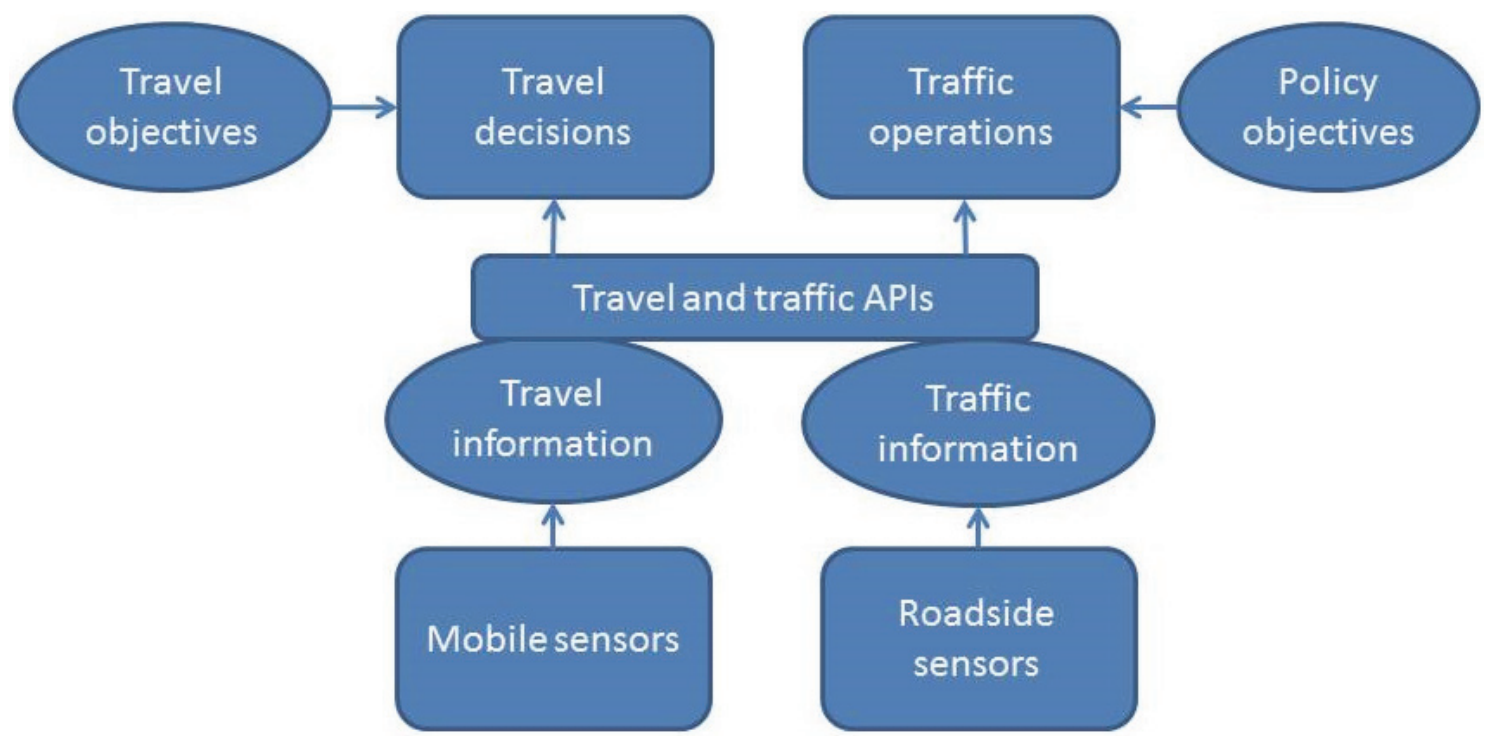

Figure 2: Scheme of integrating traffic and individual travel information on an operational level.

Surely, the usefulness of advice to the traveller would be enhanced by knowledge about (future) traffic operations (requirement 2). By the same token, the quality of traffic prediction would be increased when the intentions or likely future decisions of individual travellers are known to the traffic manager (requirement 1). Finally, without any interaction, there is no knowledge about (a change in) travellers' perceptions regarding certain traffic management measures (requirement 3 ).

In some projects such as SUNSET (Sunset, 2013) and I-zone (Veenstra et al., 2010), the information from travellers (mobile sensors) and traffic operators (roadside sensors) have been combined. This is shown schematically in Figure 2. Again, the architecture is more complicated in reality, and both projects comprise much more than combining different data sources. However, the general use of data in these projects is well captured by Figure 2 . Information derived from roadside and mobile sensor data are combined in one large database. Third parties, mostly private companies, can retrieve this information via APIs, and can use this information in all kinds of apps they develop for travellers.

The main characteristic of such an architecture is sharing of underlying sensor data, and providing these data to the larger public. However, there are two main drawbacks of such an architecture regarding DTM. First, travel information on such 


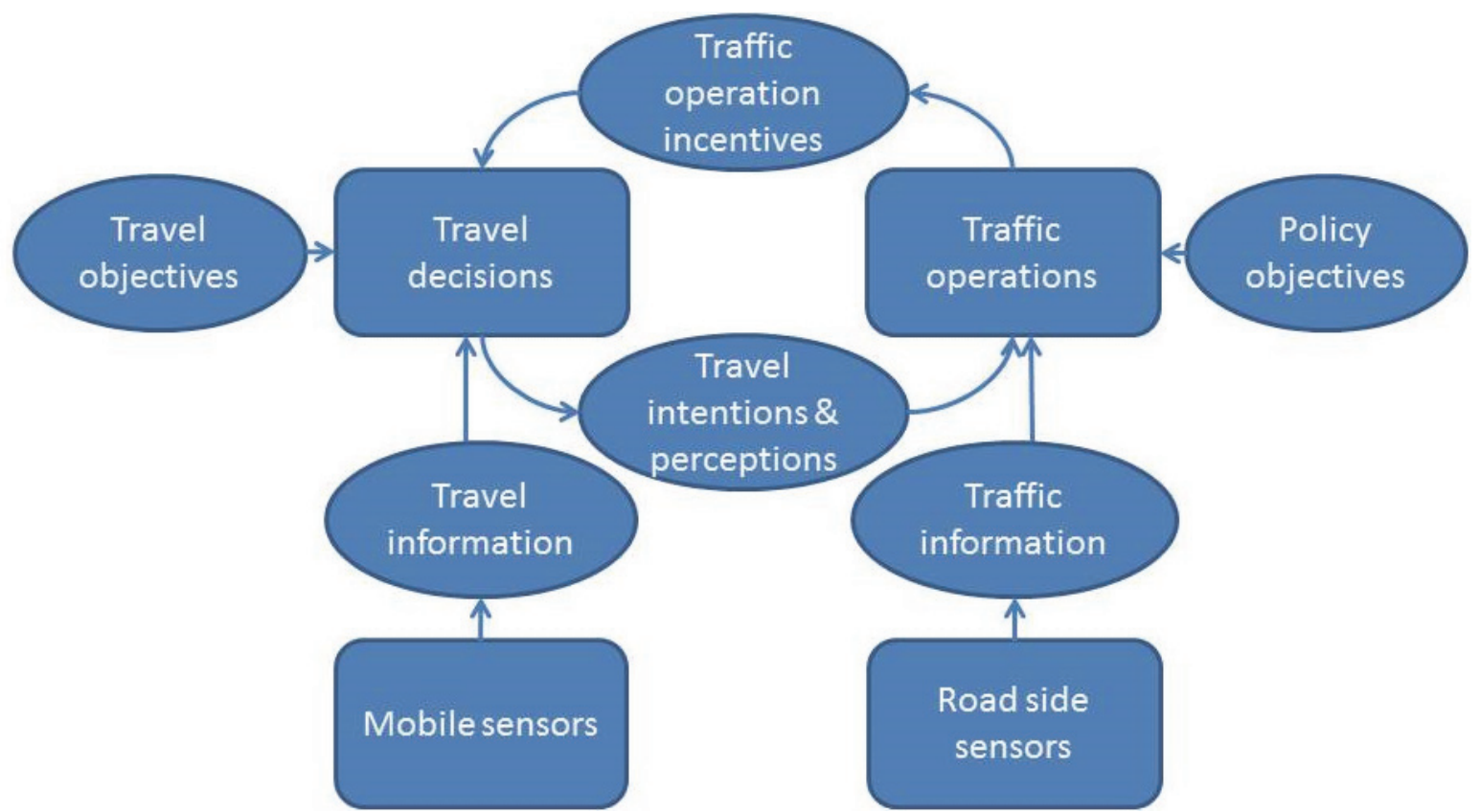

Figure 3: Scheme of integrating traffic and individual travel information on a strategic level.

an operational level is sensitive (regarding privacy) and is also regarded as quite valuable by companies that gather the data. It is therefore not very likely that these important players are willing to share their data. Secondly, these operational data do not provide the intentions of traffic operators and travellers. In fact, the main difference between roadside and mobile sensor data is not that they necessarily measure very different things (although not exactly the same either), but that they are used by very different users and for very different purposes. The real strength of sharing information would be on a higher, strategic, level such as shown in Figure 3.

In Figure 3, there is directly feedback between traffic operations and travel decision making. Based on traffic information from roadside devices and traffic policies, traffic operations are set to manage the traffic (such as in Figure1). However, information about travellers' reactions to and perceptions of certain management measures are provided to the traffic operators and are used to improve the management scenarios. At the same time, updated management scenarios are provided to travellers(' apps), enabling travellers to take specific traffic measures (including possible incentives for favourable behaviour) into account when planning their trip.

Instead of sharing traffic data, the main characteristic of this DTM vision is sharing of intentions, plans and measures between traffic operators and travellers. The corresponding architecture would connect well to the DTM requirements mentioned in section 2 . The question would then be: what kind of information is exactly shared and how is this information shared? Should the information include detailed operational data such as green times of individual traffic lights given specific inflow intensities, or should the information be provided on a more aggregated and strategic level? To answer these questions follow up research is needed, preferably in a European project with as main aim to develop such a future DTM system for main European cities.

\section{REFERENCES}

Ariely, D., 2009. Predictably Irrational - Revised and expanded edition. London: HarperCollingsPublishers.

Avineri, E., Prashker, J. N., 2004. Violations of expected utility theory in route-choice stated preferences, Transportation Research Record, vol. 1894, pp. 222229.

Bajwa, S. I., Chung, E., Kuwahara, M., 2004. An adaptive travel time prediction model based on pattern matching, In $11^{\text {th }}$ World Congress on Intelligent Transport Systems, Nagoya, Japan.

Bie, j., Bilsma, M., Broll, G., Cao, H., Hjalmarsson, A., Hodgson, F., Holleis, P., Van Houten Y., Jacobs, K., Koolwaaij, J., Kusumastuti, D., Luther, M., 2012. Move Better with tripzoom, International Journal on Advances in Life Sciences, vol. 4, no. 3\&4, pp. 125 135 
Blokpoel, R., Vreeswijk, J., 2011. Vehicle inductive profile for incident detection, In Proceedings of the 18th World Congress on Intelligent Transport Systems, Orlando, United States.

Chrobok, R., Kaumann, O., Wahle, J., Schreckenberg, M., 2004. Different methods of traffic forecasting based on real data, European Journal of Operational Research, vol. 155 , pp. $558-568$

Chung, E., 2003. Classification of traffic pattern,In Proceedings of $10^{\text {th }}$ World Congress on Intelligent Transport Systems, Madrid.

Dharia, A., Adeli, H., 2003. Neural network model for rapid forecasting of freeway link travel time, Engineering Applications of Artificial Intelligence, vol. 16, pp. 607-613.

Fan, Y., Chen, Q., Douma, F., Liao, C.-F., 2012. Smartphone-Based Travel Experience Sampling and Behavior Intervention among Young Adults. Intelligent Transportation Systems Institute, Center for Transportation Studies, University of Minnesota.

Google Maps. Maps.google.com Accessed: 2013

Hasberg, P., Serwill, D., 2000. Stadtinfoköln - a global mobility information system for the Cologne area, In $7^{\text {th }}$ World Congress on Intelligent Transport Systems, Turin, Italy.

Henkel, L. A., Mather, M., 2007. Memory attributions for choices: How beliefs shape our memories, Journal of Memory and Language, vol. 57, pp. 163-176.

Jan, O., Horowitz, A.J., Peng, Z.R., 2000. Using global positioning system data to understand variations in path choice. Transportation Research Record, vol. 1725 , pp. 37-44.

Jayakrishnan, R., Mahmassani, H. S., Hu, T. Y., 1994. An evaluation tool for advanced traffic information and management systems in urban networks, Transportation Research Part C, vol. 2, pp. 129-147.

Kellerman, A., Schmid, A., 2000. Mobinet: Intermodal traffic management in Munich -control centre development, In $7^{\text {th }}$ World Congress on Intelligent Transport Systems, Turin, Italy.

Leitsch, B., 2002. A Public-privat partnership for mobility - Traffic management Center Berlin, In $9^{\text {th }}$ World Congress on Intelligent Transport Systems, Chicago, USA.

Li, M., Dai, J., Sahu, S., Naphade, M., 2011. Trip analyzer through smartphone apps, In Proceedings of the 19th ACM SIGSPATIAL International Conference on Advances in Geographic Information Systems, ACM, 537-540.

Mahmassani, H. S., Chang, G.-L., 1987. On boundedly rational user equilibrium in transportation networks, Transportation Science, vol. 21, pp. 89-99.

Mather, M., Shafir, E., Johnson, M. K., 2003. Remembering chosen and assigned options, Memory and Cognition, vol. 31, pp. 422-433.

Nitsche, P., Widhalm, P., Breuss, S., Maurer, P., 2012. A Strategy on How to Utilize Smartphones for Automatically Reconstructing Trips in Travel Surveys. Procedia - Social and Behavioral Sciences, vol. 48, pp. 1033-1046.
Ortúzar, J. D. D., Armoogum, J., Madre, J.-L., Potier, F., 2010. Continuous Mobility Surveys: The State of Practice. Transport Reviews, vol. 31, pp. 293-312.

Papinski, D., Scott, D.M., 2009. A GIS Toolkit for route choice analysis, Journal of Transport Geography, vol. 19 , pp. $434-442$.

Rieser-Schüssler, N., Balmer, M., Axhausen, K.W., 2012. Route choice sets for very high-resolution data, Working paper Transport and Spatial Planning, EHT, Zürich, Switzerland

Schönfelder, S., Axhausen, K. W., 2010. Urban Rhythms and Travel Behaviour. Spatial and Temporal Phenomena of Daily Travel, Farnhem/Burlington, Ashgate Publishing.

Simon, H. A., 1955. A behavioural model of rational choice, Quarterly Journal of Economics, vol. 69, pp. 99-118.

Stopher, P. R., 2009. The travel survey toolkit: where to from here? Transport survey methods, keeping up with a changing world, pp. 15-46.

Stopher, P.R., Zhang, Y., 2011. The repetitiveness of daily travel, Transportation Research Board Annual Meeting, Washington DC, USA.

Sunset, http://sunset-project.eu/ Accessed: 2013

Thomas, T., Weijermars, W. A. M., Van Berkum, E. C., 2008. Variations in urban traffic volumes, European Journal of Transport and Infrastructure Research, vol. 8. no. 3, pp. 252-264.

Thomas, T., Weijermars, W. A. M., and Van Berkum, E. C., 2010. , IEEE transactions on intelligent transport systems, vol. 11, no. 1, pp. 71-80.

Tversky, A., Kahnemann, D., 1981. The framing of decisions and the psychology of choice, Science, vol. 211, pp. 453-458.

Veenstra, S.A., Teeuw, W.B., Meeuwissen, M., 2010 Incentive Zone Enschede; Het verleiden tot gedragverandering op basis van gepersonaliseerde, multi-modale mobiliteitsinformatie en -advies, Colloquium Vervoersplanologisch Speurwerk (CVS 2010), Roermond, The Netherlands.

Vreeswijk, J. D., Thomas, T., Van Berkum, E. C., Van Arem, B., 2013. Drivers' perception of route alternatives as indicator for the indifference band. In Proceedings of 92th Annual Meeting of the Transportation Board, Washington DC, USA.

Weijermars , W. A. M., 2007. Analysis of urban traffic patterns using clustering, Ph.D. Thesis, University of Twente, Enschede, The Netherlands.

Wild, D., 1997. Short-term forecasting based on a transformation and classification of traffic volume time series, International Journal of Forecasting, vol. 13 , pp. 63-72.

Yin H., Wong, S. C., Xu, J., Wong, C. K., 2002. Urban traffic flow prediction using a fuzzy-neural approach, Transportation Research Part C, vol. 10, pp. 85-98.

Zhu, S., Levinson, D., 2010. Do people use the shortest path? An empirical test of Wardrop's first principle, In Conference proceedings of the 4th International Symposium on Transportation Network Reliability, Minneapolis, USA. 